\title{
Review of: "Long term monitoring of the reproductive behavior of wild Chinese pangolin (Manis pentadactyla)"
}

yingjie song

Potential competing interests: The author(s) declared that no potential competing interests exist.

1. Some burrows were located in farmland, whether to consider the influences of farmland farming behavior on the selection of burrows' location. It may be interesting.

2. If the courtship, mating and hatching behavior of pangolins is observed, this study will be more meaningful. 